\title{
CAFs enhance paclitaxel resistance by inducing EMT through the IL-6/JAK2/STAT3 pathway
}

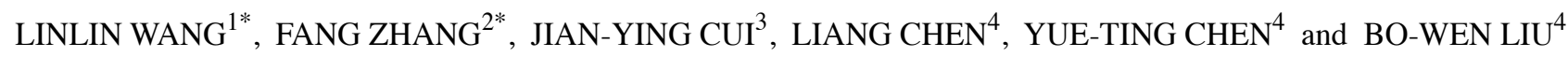 \\ ${ }^{1}$ Department of Radiation Oncology, Shandong Cancer Hospital Affiliated to Shandong University, \\ Shandong Academy of Medical Sciences, Jinan, Shandong 250117; \\ ${ }^{2}$ Department of Radiology, Provincial Hospital Affiliated to Shandong University, Jinan, Shandong 250021; \\ ${ }^{3}$ Department of Obstetrics and Gynecology, Jiyang Public Hospital, Jinan, Shandong 251400; \\ ${ }^{4}$ Department of Gynecologic Oncology, Shandong Cancer Hospital Affiliated to Shandong University, \\ Shandong Academy of Medical Sciences, Jinan, Shandong 250117, P.R. China
}

Received October 20, 2017; Accepted March 9, 2018

DOI: 10.3892/or.2018.6311

\begin{abstract}
Carcinoma-associated fibroblasts (CAFs) are the major components of mesenchymal cells in the inflammatory tumor microenvironment. They are involved in epithelial-mesenchymal transition (EMT) and chemotherapy resistance by directly contacting with cancer cells or secretory cytokines. In the present study, we examined the role of CAFs in the induction of EMT in ovarian cancer. Primary ovarian cancer cells, CAFs and normal fibroblasts (NFs) were isolated from fresh cancer tissue and cultured for immunohistochemistry studies. Enzyme-linked immunosorbent assay (ELISA) was used to detect the expression of IL- 6 in the culture supernatants of these cells. The expression of IL-6 at the mRNA level was examined by RT-PCR. The expression of IL-6 at the protein level in ovarian cancer tissues was determined using an immunofluorescence assay in both tissue sections and cell lobes. OVCAR3 cells were treated with the culture supernatants collected from CAFs and NFs. IL-6 monoclonal antibody (mAb) was employed to neutralize IL-6. The expression of phosphorylated STAT3 was assessed. Changes in EMT, proliferation, invasion and proapoptotic protein expression were also examined. Flow cytometry was performed to detect the changes in apoptosis resistance of OVCAR3 cells. The JAK2/STAT3 pathway-specific inhibitor AG490 was used to block this pathway and the $\beta$-TGF inhibitor was used to inhibit EMT. The clinical data of patients treated in our hospital were
\end{abstract}

Correspondence to: Dr Liang Chen, Department of Gynecologic Oncology, Shandong Cancer Hospital Affiliated to Shandong University, Shandong Academy of Medical Sciences, 107 Jiyan Road, Jinan, Shandong 250017, P.R. China

E-mail: 155338644@qq.com

*Contributed equally

Key words: CAFs, EMT, paclitaxel resistance, IL-6 collected between January 1st, 2009 and June 30th, 2013. The expression of interstitial IL-6 in paraffin-embedded tissues was detected by immunohistochemistry. The relationship between the expression of interstitial IL- 6 and the treatment response was examined by linear regression and multiple linear regression analyses. We found that CAFs were the main source of IL- 6 in ovarian cancer tissue. CAFs promoted the phosphorylation of STAT3 in ovarian cancer and enhanced the proliferation, invasion and EMT. Enhanced EMT may lead to apoptosis resistance, inhibitory expression of pro-apoptotic proteins and paclitaxel resistance. A total of 255 patients were enrolled in this retrospective study. Univariate and multivariate analyses revealed that age, CA125, interstitial IL-6 expression and cytoreduction satisfaction were closely related to the sensitivity of the TP (docetaxel plus cisplatin or carbopatin) regimen in ovarian cancer $(\mathrm{P}<0.05)$. These results demonstrated that CAFs highly secreted IL- 6 and promoted $\beta$-TGF-mediated EMT in ovarian cancer via the JAK2/STAT3 pathway, leading to inhibited apoptosis and subsequent paclitaxel resistance. Therefore, CAFs may be a new therapeutic target for the treatment of ovarian cancer.

\section{Introduction}

Epithelial ovarian cancer (EOC) is the third most common type of cancer of the female reproductive system in the USA. EOC has the highest mortality rate among the malignancies of the female reproductive system with a 5-year survival rate of only $\sim 30-40 \%$ in the USA (1). EOC is characterized by intra-abdominal invasion and metastasis, and EOC patients in advanced clinical stages often have multiple distant metastases. The recurrence rate of ovarian cancer is high and cancer cells may develop paclitaxel resistance after relapse, resulting in chemotherapy failure. Therefore, it is important to inhibit metastasis and chemotherapy resistance of ovarian cancer. Epithelial-mesenchymal transition (EMT) has been demonstrated to enhance epithelial cancer cells with the following three malignant potentials (2,3): i) Invasion and metastasis, ii) resistance to apoptosis and iii) acquisition of stem cell 
properties. However, it remains unclear whether reversing EMT can inhibit invasion and metastasis and ameliorate the drug resistance of ovarian cancer.

The tumor microenvironment consists of tumor cells, fibroblasts, smooth muscle cells, nerves, blood vessels and a variety of inflammatory/immune cells $(4,5)$. These cells and inflammatory cytokines constitute the inflammatory tumor microenvironment, which may promote the proliferation and metastasis of tumor. As the main components of mesenchymal cells in tumor microenvironment, fibroblasts are known as tumor-associated fibroblasts (CAFs). They are activated fibroblasts in the tumor stroma, which express $\alpha$-smooth muscle actin ( $\alpha$-SMA) and exhibit the characteristics of myofibroblasts (6).

It has been demonstrated that CAFs secrete various inflammatory cytokines via an autocrine or paracrine mechanism, such as IL-6, COX-2, CXCL12 and HIF-1 $\alpha$ (4,7,8). CAFs can also regulate the sensitivity of tumor cells to chemotherapy, playing important roles in improving the efficacy of chemotherapy and reversing drug resistance. Johansson et al (9) have found that co-culture of CAFs and head and neck squamous cell carcinoma (HNSCC) cells can upregulate the expression of MMP-1, thereby decreasing the sensitivity of HNSCC cells to cephalosporin. Yu et al (10) have found that miR21 is transferred from cancer-associated adipocytes (CAAs) or CAFs to cancer cells, where it suppresses apoptosis in ovarian cancer cells and induces chemoresistance by binding to its direct novel target, APAF1.

As an important inflammatory factor, interleukin-6 (IL-6) binds to its receptor IL-6R on the cell membrane and activates several downstream pathways, such as the JAK2/STAT3 and P13K/AKT pathways. The JAK2/STAT3 pathway is a signal transduction pathway from the membrane to the nucleus. The activation of JAK2 protein kinase can catalyze the phosphorylation of STAT3 protein into the nucleus, which can regulate the expression of EMT-related genes and other genes $(11,12)$. At present, it has been found that the overactivation of the IL-6/JAK2/STAT3 pathway can promote the EMT of tumor cells (13). Recent studies indicated that EMT is closely associated with chemotherapy resistance by promoting apoptosis resistance $(14,15)$. In the present study, we aimed to investigate the effect of CAF-derived IL- 6 on EMT in ovarian cancer cells via the JAK2/STAT3 pathway. Written informed consent was obtained from all the patients prior to treatment. This study was approved by the Ethics Committee of Shandong Cancer Hospital Affiliated to Shandong University. Our findings further elucidated the role of CAFs in the development of chemotherapy resistance in the tumor microenvironment.

\section{Materials and methods}

Reagents and antibodies. The JAK2/STAT3 pathway inhibitor AG490 was purchased from APExBIO (Apexbio Technology LLC, Houston, TX, USA) and the $\beta$-TGF inhibitor SB431542 was obtained from Selleck Chemicals (Houston, TX, USA). Mouse anti-IL6 (cat. no. MAB206) was purchased from R\&D Systems, Inc. (Minneapolis, MN, USA). The rabbit anti- $\alpha$-SMA (cat. no. 55135-1-AP) and rabbit anti-Vimentin (cat.no. 10366-1-AP) antibodies were supplied from Proteintech
Group, Inc. (Chicago, IL, USA). Other primary antibodies were provided by Abcam (Cambridge, MA, USA), including anti-E-cadherin antibody (cat. no. ab40772), anti-N-cadhein antibody (cat.no.ab76011), anti-Bax antibody (cat.no.ab32503), anti-Bcl-2 antibody (cat. no. ab32124), anti-caspase-3 antibody (cat. no. ab13847). Alexa Fluor 594-conjugated goat anti-rabbit IgG (H+L) (cat. no. SA00006-4), Alexa Fluor 488-conjugated AffiniPure goat anti-mouse IgG (H+L) (cat. no. SA00006-4) and HRP-conjugated AffiniPure goat anti-rabbit IgG $(\mathrm{H}+\mathrm{L})$ (cat. no. SA00001-2) or mouse IgG (H+L) (cat. no. SA00001-1) were purchased from Proteintech Group. The other reagents were obtained from Sigma-Aldrich (Merck KGaA, Darmstadt, Germany).

Cell culture. The human ovarian cancer cell line OVCAR3 was purchased from the cell bank of the Chinese Academy of Sciences (Shanghai, China). The cells were maintained in RPMI-1640 medium supplemented with $10 \%$ fetal bovine serum (FBS; Gibco; Thermo Fisher Scientific, Inc., Waltham, MA, USA), $100 \mathrm{U} / \mathrm{ml}$ penicillin and $100 \mathrm{mg} / \mathrm{ml}$ streptomycin at $37^{\circ} \mathrm{C}$ in a humidified atmosphere containing $5 \% \mathrm{CO}_{2}$. The cells in the logarithmic growth phase were used for further experiments.

Isolation and confirmation of human ovarian fibroblasts. Intraoperative cleaning was performed to cultivate CAFs. Briefly, tissues were washed with cleaning buffer (PBS with penicillin-streptomycin) and the epithelial and adipose tissues were removed. The remaining connective tissues were cut into $1 \times 1 \times 1 \mathrm{~mm}$ pieces. The fragments were cultured in DMEM supplemented with 5\% FBS and $1 \%$ antibiotic-antimycotic solution and incubated at $37^{\circ} \mathrm{C}$ in a humidified atmosphere containing $5 \% \mathrm{CO}_{2}$. The culture medium was refreshed every 2 or 3 days. After the cellular fusion of the cells, the cell passage ratio was 1:2. The third-generation cells were used for verification.

Normal ovarian specimens removed during myomectomy were selected to cultivate normal fibroblasts (NFs) following the above-mentioned steps. Fresh ovarian cancer tissue was used for the primary culture of ovarian cancer cells. Since fibroblasts are easier to adhere than endothelial cells, most fibroblasts were removed by incubating the cells at different durations.

To confirm CAFs and NFs, the isolated cells grown on slides were fixed with $4 \%$ paraformaldehyde for $20 \mathrm{~min}$ and then washed with PBS. The cells were then blocked with $10 \%$ normal goat serum for $1 \mathrm{~h}$. Subsequently, the slides were incubated with primary antibodies against $\alpha$-SMA (1:100) or Vimentin $(1: 100)$ at $37^{\circ} \mathrm{C}$ for $1 \mathrm{~h}$, followed by incubation with HRP-conjugated secondary antibody at $37^{\circ} \mathrm{C}$ for $1 \mathrm{~h}$. Finally, the cells were stained with a DAB substrate (Abcam).

Dual immunofluorescence staining. Paraffin-embedded sections of ovarian cancer tissues were obtained in a routine manner. Slides were deparaffinized in xylene and serially rehydrated with alcohol and water, and then epitope retrieval was performed by heating the sections in a microwave oven. The sections were then blocked in $10 \%$ normal goat serum for $1 \mathrm{~h}$ and incubated with primary antibodies against $\alpha$-SMA $(1: 100)$ or IL-6 (1:100) at room temperature for $1 \mathrm{~h}$. They were 
then incubated in Alexa Fluor 594-conjugated goat anti-rabbit IgG $(\mathrm{H}+\mathrm{L})$ and Alexa Fluor 488-conjugated AffiniPure goat anti-mouse $\operatorname{IgG}(\mathrm{H}+\mathrm{L})$ secondary antibodies for $30 \mathrm{~min}$. Subsequently, the sections were washed with PBS, counterstained with DAPI (Abcam) at room temperature for $10 \mathrm{~min}$ and mounted using fluorescent mounting medium to protect the specimens.

For the dual immunofluorescence staining of CAFs and NFs grown on chamber slides, the cells were fixed with $4 \%$ paraformaldehyde for $20 \mathrm{~min}$ and then washed with PBS. Subsequently, the cells were blocked with $10 \%$ normal goat serum for $30 \mathrm{~min}$ and the slides were sequentially reacted with primary and secondary antibody as above described. Following counterstaining with DAPI, the slides were sealed with fluorescent mounting medium and then examined using a fluorescence microscope.

Enzyme-linked immunosorbent assay (ELISA). The expression of IL-6 secreted by cancer cells or fibroblasts was evaluated using ELISA. The cells were seeded into six-well plates at a density of $2 \times 10^{5} / \mathrm{ml}$ and cultured for $48 \mathrm{~h}$. Subsequently, culture supernatants were collected and centrifuged at $12,000 \mathrm{x} \mathrm{g}$ for $10 \mathrm{~min}$ and the IL-6 level was determined using an IL-6 ELISA kit (human Quantikine ${ }^{\circledR}$; R\&D Systems) according to the manufacturer's instructions.

RNA isolation and quantitative real-time PCR. Total RNA was isolated using an Ultrapure RNA kit (CW Biotech, Beijing, China) according to the manufacturer's instructions. Then $1 \mu \mathrm{g}$ of purified RNA was reverse transcribed into first-strand cDNA using the HiFiScript cDNA Synthesis kit (CW Biotech) and the IL-6 expression at the mRNA level was determined using UltraSYBR Mixture real-time PCR (CW Biotech). $\beta$-actin was selected as the housekeeping gene and the specific primers were synthesized by Genewiz (Beijing, China) with the sequences as follows: IL-6 forward, 5'-GTCCAGTTGCCTTCTCCC-3' and reverse, 5'-GCCTCT TTGCTGCTTTCA-3'; $\beta$-actin forward, 5'-CCCGAGCCG TGTTTCCT-3' and reverse, 5'-GTCCCAGTTGGTGACGAT GC-3'. The relative expression of IL6 was calculated using the $2^{-\Delta \Delta \mathrm{Ct}}$ method (16) and each experiment was performed in triplicate.

Western blotting. After treatment for $48 \mathrm{~h}$, the cells were harvested and lyzed in RIPA buffer and the protein concentration was determined using the BCA kit (CW Biotech). Equal amounts of total protein were separated by SDS-polyacrylamide gel electrophoresis (SDS-PAGE) and then electro-transferred onto a polyvinylidene fluoride (PVDF) microporous membrane (Millipore; Merck KGaA, Darmstadt, Germany). The membranes were incubated with primary antibodies at $4^{\circ} \mathrm{C}$ overnight, followed by incubation with secondary antibodies at room temperature for $1 \mathrm{~h}$. The blots were visualised by ECL chemiluminescence (Millipore; Merck KGaA).

Invasion assays. The invasion assays were performed using chambers with polycarbonate filters $(8-\mu \mathrm{m}$ pore size; BD Biosciences, Franklin Lakes, NJ, USA). First, $200 \mu \mathrm{l}$ cell suspension containing $3 \times 10^{5}$ cells was seeded into the upper chamber of the insert pre-coated with Matrigel (1:8 dilution; BD Biosciences). The cells were maintained in medium without serum and medium supplemented with $10 \%$ FBS was used as a chemoattractant in the lower chamber. After $24 \mathrm{~h}$ of incubation, the cells that did not invade through the membrane were wiped off using a cotton swab. Subsequently, the membranes were fixed with $4 \%$ paraformaldehyde for $30 \mathrm{~min}$ and stained with $0.5 \%$ crystal violet. The penetrating cells were quantified on five randomly selected fields.

Cell Counting Kit (CCK)-8 assay. Following the treatment of the OVCAR3 cells with co-culture supernatant or the transfection with IL-6 shRNA for $24 \mathrm{~h}$, equal numbers of cancer cells $\left(6 \times 10^{2}\right.$ cells) were incubated in 96-well plates with $100 \mu \mathrm{l}$ medium for 24, 48 and $72 \mathrm{~h}$. The number of cells was estimated using a CCK- 8 assay. Briefly, $10 \mu \mathrm{l} \mathrm{CCK-8}$ was added to each well and the cells were incubated for $1 \mathrm{~h}$. The absorbance was determined at a wavelength of $450 \mathrm{~nm}$.

Flow cytometry. The cells were transfected with IL-6 shRNA or treated with co-culture supernatant for $48 \mathrm{~h}$, followed by treatment with paclitaxel $(30 \mathrm{nM})$ for $24 \mathrm{~h}$. Subsequently, the cells were stained with PI and FITC-labelled Annexin-V and analyzed using flow cytometry (BD Biosciences). The cells that were negative for PI, but positive for FITC were considered early apoptotic cells, whereas the cells that were positive for both PI and FITC were considered late apoptotic or necrotic cells. Flow cytometry data were analyzed using the FlowJo software (BD Biosciences).

Immunohistochemical, clinical and pathological data analyses. Between January 1st, 2009 and June 30th, 2014, 269 patients underwent initial treatment at the Department of Gynecologic Oncology of Shandong Cancer Hospital, Affiliated to Shandong University. Patients with FIGO stage IIA-IV ovarian cancer underwent cytoreductive surgery (the scope of surgery included uterus, double accessory, omentum, appendix with or without pelvic or abdominal metastases). Complete clinical data of the patients and pathological paraffin blocks were obtained. Follow-up was performed using the following three methods: Medical records, telephone follow-up and outpatient review. The deadline of the follow-up was June 30th, 2016.

Two observers determined the results based on the expression of interstitial IL-6. For the reading of the pathological sections, a positive result was determined when the cytoplasm and nucleus of the fibroblasts were stained yellow in the stroma of cancer tissues. The proportion of positive cells was assessed by five visual fields, without considering the staining intensity. The expression of IL-6 was divided into two grades: High expression group, in which at least $50 \%$ of stromal fibroblasts was positive; low expression group, in which the percentage of positive stromal fibroblasts was $<50 \%$.

Statistical analysis. All statistical analyses were performed using the SPSS version 17.0 software (SPSS, Inc., Chicago, IL, USA). The relationship between the expression of interstitial IL-6 and other clinical features and treatment responses was examined by linear regression and multiple linear regression analysis. 
A

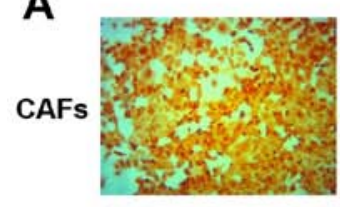

NFs

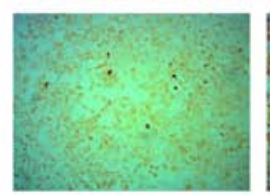

a-SMA
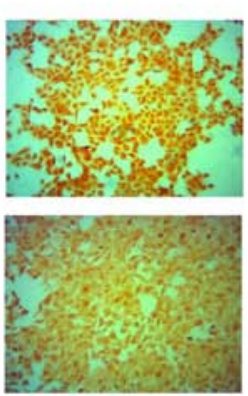

Vimentin

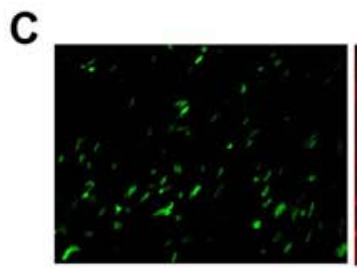

IL-6

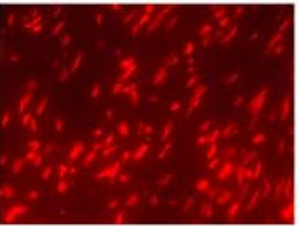

a-SMA

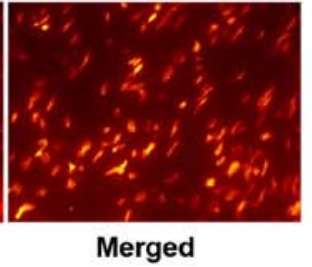

Merged
B

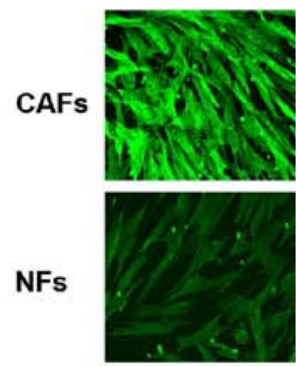

IL-6

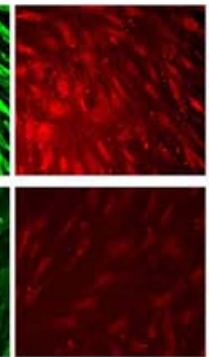

a-SMA

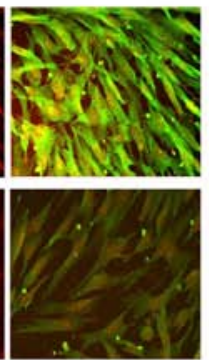

Merged
D

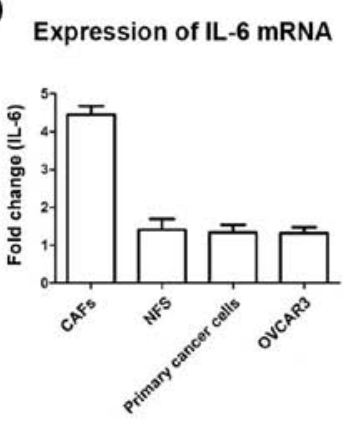

E

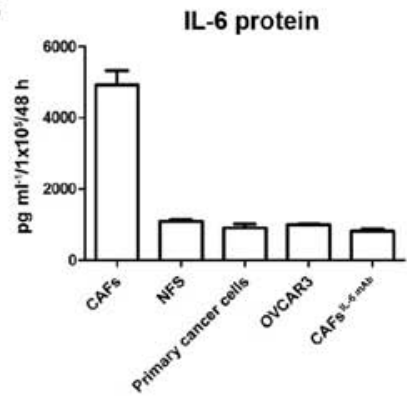

Figure 1. CAFs are the main source of IL-6 secretion. (A) Following the separation and purification of CAFs and NFs, the cells were stained with $\alpha-$ SMA (100-fold) and Vimentin (100-fold). Both CAFs and NFs expressed Vimentin. However, $\alpha$-SMA was almost not expressed in NFs, while it was overexpressed in CAFs. (B) CAFs and NFs were stained with IL-6 and $\alpha$-SMA (100-fold). The expression of IL-6 and $\alpha$-SMA was positive in CAFs. The expression of IL-6 and $\alpha$-SMA detected in NFs was weak. (C) Immunofluorescence staining of IL-6 (100-fold) and $\alpha$-SMA (100-fold) was performed on histological sections of EOC. Similarly, these results revealed that IL- 6 was mainly expressed in the interstitium and interstitial $\alpha$-SMA-labelled CAFs. (D) The culture supernatants of CAFs, NFs, primary cancer cells and OVCAR3 cells were extracted to detect the expression of IL-6 by ELISA. The expression of IL-6 in CAFs was significantly higher than that in NFs, primary cancer cells and OVCAR3. (E) Detected by RT-PCR, the expression of IL-6 at the mRNA level in CAFs was significantly higher than that in NFs, primary cancer cells and OVCAR3.

\section{Results}

Expression of IL-6 in ovarian cancer tissue and its localization . Purified CAFs and NFs were identified by immunofluorescent staining using two antibodies as follows: i) Anti-Vimentin antibody, which is often used for the identification of fibroblasts and ii) anti- $\alpha$-SMA antibody, which is a marker of activated fibroblasts. The expression of Vimentin was detected in both CAFs and NFs. However, $\alpha$-SMA was almost not expressed in NFs, while it was overexpressed in CAFs (Fig. 1A). This finding indicated that fibroblasts in the tumor stroma were activated, which are called 'cancer associated fibroblasts'.

Subsequently, we detected the expression of IL-6 in CAFs and NFs. IL-6 was highly expressed in CAFs expressing the activity marker $\alpha$-SMA. Furthermore, $\alpha$-SMA and IL- 6 were co-localized in CAFs. In contrast, the expression of IL-6 was weak in NFs with sparse $\alpha$-SMA expression (Fig. 1B). Similarly, IHC staining of ovarian epithelial carcinoma revealed that IL- 6 was also mainly expressed in interstitial cells of ovarian cancer and co-localized with $\alpha$-SMA in interstitial CAFs (Fig. 1C). This finding indicated that CAFs were the main source of IL-6 secretion in ovarian cancer.

Since IL-6 is a secretory protein, we further assessed the expression of IL-6 in the culture supernatants of CAFs, NFs, primary cancer cells and OVCAR3 cells using an ELISA kit. The expression of IL-6 in CAFs was significantly higher than that in NFs, primary cancer cells and OVCAR3 (Fig. 1D). Detected by RT-PCR, the expression of IL-6 mRNA in CAFs was significantly higher than that in NFs, primary cancer cells and OVCAR3 (Fig. 1E). This result also revealed that the secretion of IL- 6 in ovarian cancer was mainly from interstitial CAFs.

CAF-derived IL-6 promotes the proliferation, migration and EMT of ovarian cancer cells. OVCAR3 cells were incubated with the culture supernatants collected from CAFs and NFs. The CCK- 8 proliferation assay revealed that the culture supernatant collected from CAFs significantly enhanced the proliferation of OVCAR3 cells (Fig. 2A). Similarly, the invasive potential of OVCAR3 cells treated with culture supernatant from CAFs was significantly higher than that of cells treated with culture supernatant from NFs (Fig. 2B). However, with the addition of IL- $6 \mathrm{mAb}$, the effect of CAF supernatant on the proliferation and invasion of OVCAR3 cells was significantly suppressed (Fig. 2A and B). This finding indicated that CAF-derived IL-6 was an important factor in promoting the proliferation and invasion of the OVCAR3 cells.

EMT is an important step through which ovarian cancer cells obtain malignant biological behavior $(12,17)$. Subsequently, we examined the EMT markers of OVCAR3 cells after the treatment of the culture supernatant of CAFs. The results revealed that the culture supernatant of CAFs 
A

Proliferation of OVCAR3 cells treated with different supernatants

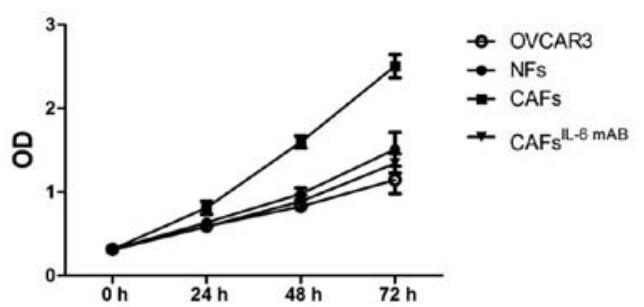

B

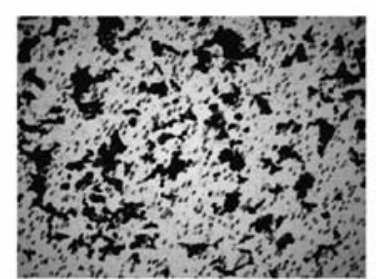

OVCAR3

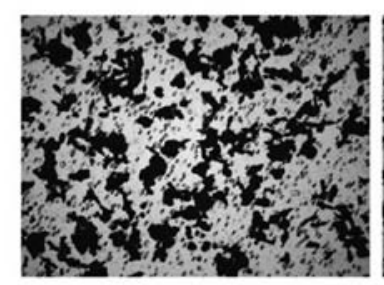

CAFs

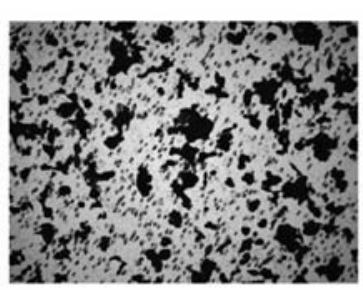

NFs

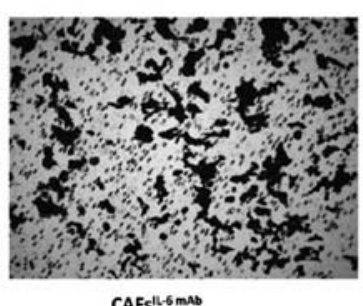

C

E-cadherin

$\mathrm{N}$-cadherin

$\beta$-catenin

Vimentin

GAPDH
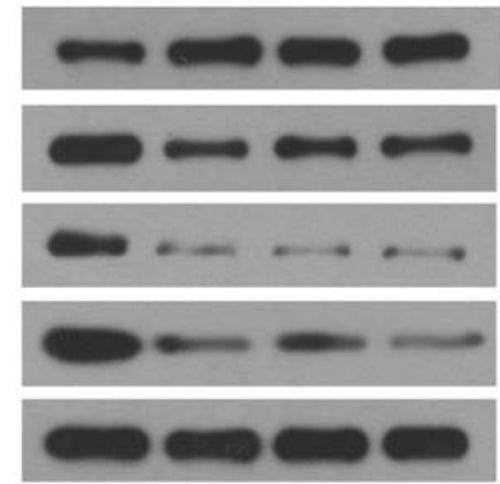

CAFs NFs OVCAR3 CAFs 1 -6 mab

Figure 2. CAF-derived IL-6 promotes the proliferation, migration and EMT of ovarian cancer cells. The culture supernatants of CAFs and NFs were collected and applied to OVCAR3 cells. (A) CCK-8 proliferation assay revealed that the supernatant derived from CAFs could significantly enhance the proliferation of OVCAR3 cells. Similarly, the invasive potential of OVCAR3 cells treated with CAF supernatant was significantly higher than that of NF supernatant. However, with the addition of IL- $6 \mathrm{mAb}$, the effect of CAF supernatant on the proliferation and invasion of OVCAR3 cells was significantly reduced. (B) The effect of CAF supernatant on the expression of EMT markers was significantly attenuated by the addition of IL- 6 mAb. There was no significant change in EMT markers after treatment with NFs supernatant. (C) Western blot analysis revealed that the OVCAR3 cells had significantly increased expression of the interstitial markers N-cadherin and Vimentin, and decreased expression of the epithelium marker E-cadherin, suggesting that the EMT of OVCAR3 cells was significantly enhanced.

significantly upregulated interstitial markers $\mathrm{N}$-cadherin and Vimentin and decreased the expression of epithelium marker E-cadherin in OVCAR3 cells, indicating that the EMT of the OVCAR3 cells was significantly enhanced. However, the effect of CAF supernatant on the expression of EMT markers in OVCAR3 cells was significantly attenuated by the addition of IL-6 mAb. There were no significant changes in the EMT markers of OVCAR3 cells after treatment with NF supernatant (Fig. 2C), indicating that CAF-derived IL-6 promoted the EMT of OVCAR3 cells.

The JAK2/STAT3 pathway is required for regulating EMT in ovarian cancer cells induced by $I L-6$. In the present study, we treated OVCAR3 cells with culture supernatants of CAFs and NFs. The phosphorylation levels of JAK2 and STAT3 proteins in OVCAR3 cells treated with culture supernatant from CAFs were significantly higher than those of cells treated with culture supernatant from NFs. Furthermore, after the addition of IL-6 mAb, the phosphorylation levels of JAK2 and STAT3 proteins were decreased (Fig. 3A).

AG490, the JAK2/STAT3 signaling pathway specific inhibitor, was applied to OVCAR3 cells treated with the culture supernatant from CAFs. Subsequently, the expression of interstitial markers $\mathrm{N}$-cadherin and Vimentin were decreased and the expression of epithelium marker E-cadherin was increased in OVCAR3 cells. This result indicated that CAF-derived IL-6 mediated the EMT in OVCAR3 cells via the JAK2/STAT3 pathway (Fig. 3B).

CAF-derived IL- 6 enhances paclitaxel resistance of ovarian cancer cells via cellular EMT. OVCAR3 cells were treated with the culture supernatants collected from CAFs and NFs. After $24 \mathrm{~h}$, the cells were treated with paclitaxel to induce apoptosis, which was confirmed by flow cytometry. We found that the number of apoptotic cells was decreased in OVCAR3 cells treated with CAF supernatant. After the addition of IL-6 mAb, paclitaxel resistance was reduced and paclitaxel-induced apoptosis was promoted (Fig. 4A). Furthermore, we assessed the expression of apoptotic-related protein in OVCAR3 cells by western blot analysis. Our results indicated that the expression of pro-apoptotic protein was decreased and the expression of apoptosis-suppressing protein was enhanced in cells treated with CAF supernatant compared with the cells treated with NF supernatant. As displayed in 

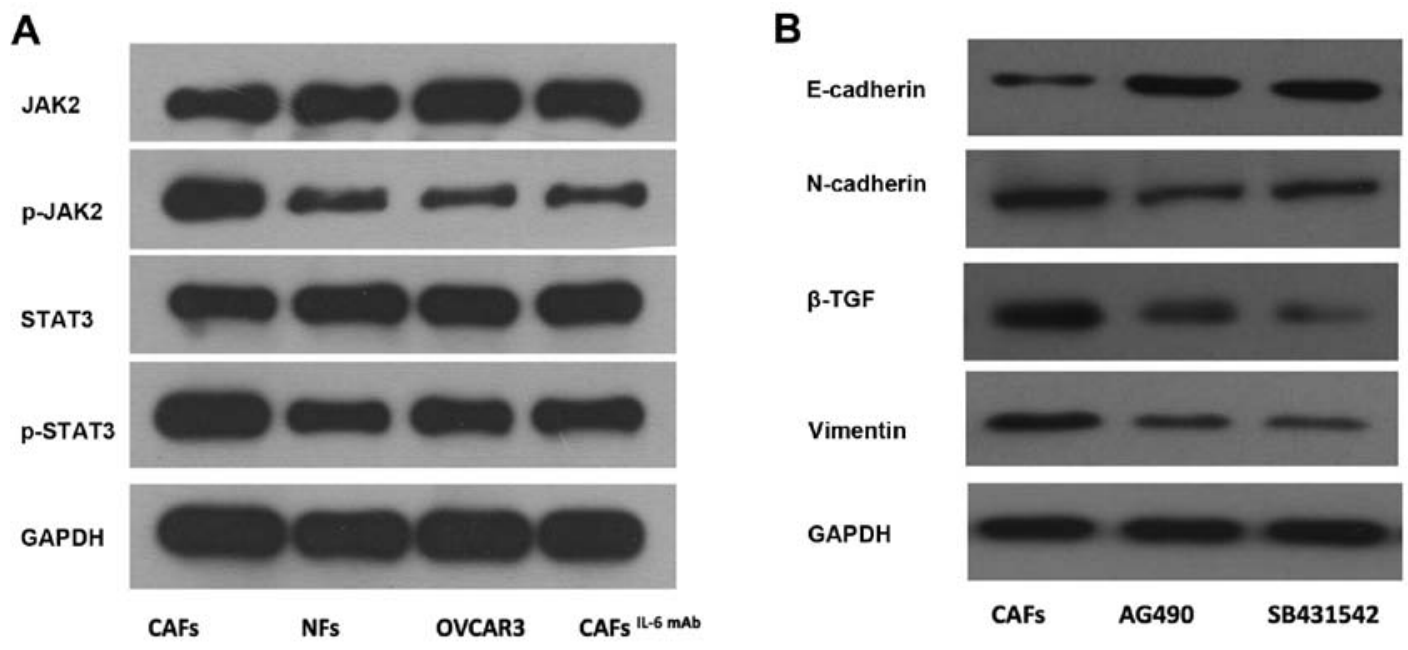

Figure 3. The JAK2/STAT3 pathway is required for regulating EMT in ovarian cancer cells induced by IL-6. (A) The culture supernatants of CAFs and NFs were applied to OVCAR3 cells. The phosphorylation levels of JAK2 and STAT3 in OVCAR3 cells treated with CAF supernatant were significantly higher than those in cells treated with NF supernatant. After the addition of IL- $6 \mathrm{mAb}$, the phosphorylation levels of JAK2 and STAT3 were decreased. (B) After the JAK2/STAT3-signaling-pathway-specific inhibitor AG490 was added, the expression of the interstitial markers N-cadherin and Vimentin was decreased and the expression of the epithelium marker E-cadherin was increased. These results indicated that CAF-derived IL-6 could mediate EMT in OVCAR3 cells via the JAK2/STAT3 pathway.

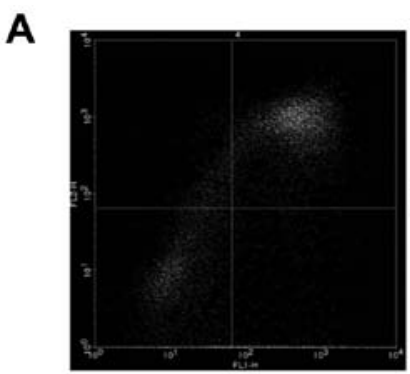

OVCAR3

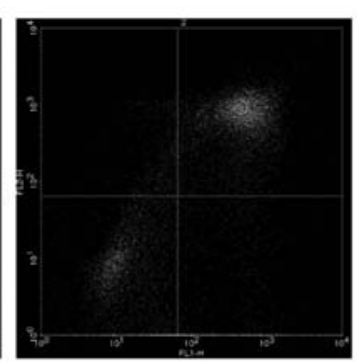

NFs

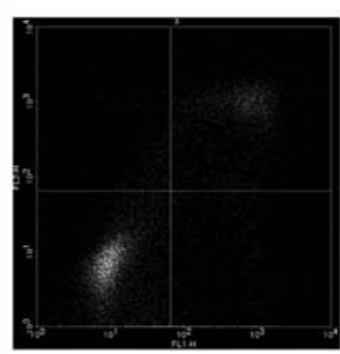

CAFs

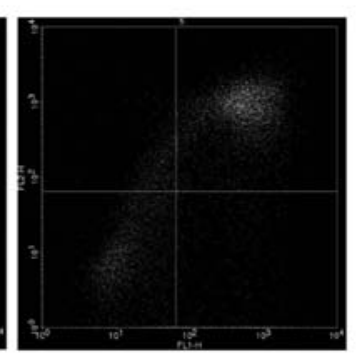

CAFs ${ }^{1-6}$ mAb

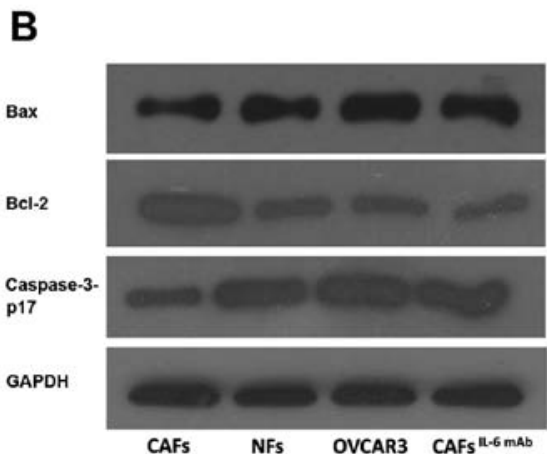

C

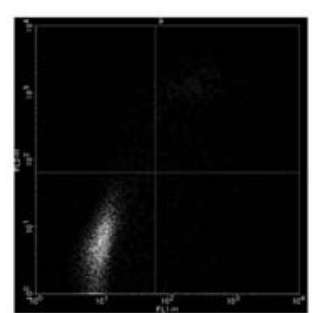

CAFs

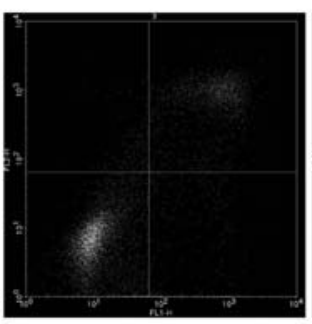

AG490

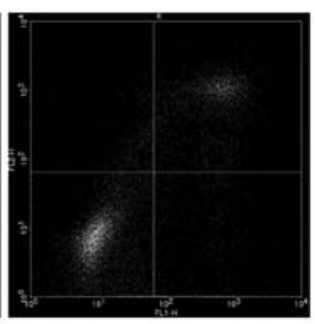

SB431542

Figure 4. CAF-derived IL-6 enhances paclitaxel resistance of ovarian cancer cells through cellular EMT. (A) The culture supernatants of CAFs and NFs were applied to OVCAR3 cells. The number of apoptotic cells was decreased in OVCAR3 cells treated with CAF supernatant. After the addition of IL-6 mAb, paclitaxel resistance was reduced and paclitaxel-induced apoptosis was promoted. (B) The expression of pro-apoptotic protein Bax and caspase-3-p17 was decreased, and the expression of apoptosis-suppressing protein Bcl-2 was enhanced in cells treated with CAF supernatant compared with NF supernatant. (C) The number of apoptotic cells treated with paclitaxel was increased after the addition of SB431542 and AG490.

Fig. 4B the addition of IL-6 mAb neutralized the expression of these genes, indicating that CAF-derived IL-6 was involved in paclitaxel resistance of OVCAR3 cells.

SB431542, an inhibitor of TGF- $\beta$, was applied to the culture system. Furthermore, EMT in OVCAR 3 cells treated with CAF supernatant was inhibited, resulting in the downregulation of the interstitial markers $\mathrm{N}$-cadherin and Vimentin as well as the upregulation of epithelium marker E-cadherin (Fig. 3B). Furthermore, the number of apoptotic cells treated with paclitaxel was increased after the addition of SB431542 and AG490 (Fig. 4C). These findings indicated that CAFs could induce the EMT of cancer cells via the IL-6/JAK2/STAT3 pathway, resulting in increased apoptosis resistance, decreased expression of the pro-apoptotic protein Bax and caspase-3-p17, 
Table I. Baseline characteristics of patients.

\begin{tabular}{|c|c|}
\hline Factors & $n(\%)$ \\
\hline \multicolumn{2}{|c|}{ Age at diagnosis (years) } \\
\hline$\leq 50$ & $63(24.7)$ \\
\hline$>50$ & $192(75.3)$ \\
\hline \multicolumn{2}{|c|}{ Pathological type } \\
\hline Serous & $205(80.4)$ \\
\hline Mucus & $5(2.0)$ \\
\hline Mixed & $25(9.8)$ \\
\hline Special types $^{\mathrm{a}}$ & $20(7.8)$ \\
\hline \multicolumn{2}{|l|}{ Tumor grade } \\
\hline G1 & $73(28.6)$ \\
\hline $\mathrm{G} 2$ & $33(13.0)$ \\
\hline G3 & $149(58.4)$ \\
\hline \multicolumn{2}{|l|}{ Tumor stage } \\
\hline II & $32(12.5)$ \\
\hline III & $193(75.7)$ \\
\hline IV & $30(11.8)$ \\
\hline \multicolumn{2}{|l|}{ CA125 } \\
\hline$\leq 500$ & $158(62.0)$ \\
\hline$>500$ & $97(38.0)$ \\
\hline \multicolumn{2}{|l|}{ Interstitial IL-6 } \\
\hline Weak & $176(69.0)$ \\
\hline Strong & $79(31.0)$ \\
\hline \multicolumn{2}{|c|}{ Neoadjuvant chemotherapy } \\
\hline Yes & $224(87.8)$ \\
\hline No & $31(12.2)$ \\
\hline \multicolumn{2}{|l|}{ Cytoreduction } \\
\hline Satisfied & $176(69.0)$ \\
\hline Unsatisfied & $79(31.0)$ \\
\hline \multicolumn{2}{|c|}{ Chemosensitivity } \\
\hline Resistant & $95(37.3)$ \\
\hline Sensitive & $106(62.7)$ \\
\hline
\end{tabular}

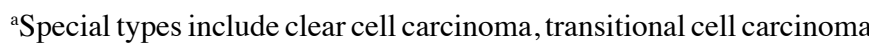
and carcinosarcoma.

and increased expression of the apoptosis-suppressing protein Bcl-2 (Fig. 4B). Finally, it may lead to pactitaxel resistance.

The expression of IL-6 in tumor stroma is related to the sensitivity of TP (docetaxel plus cisplatin or carbopatin) chemotherapy in ovarian cancer. Between January 2009 and June 2013, 255 patients underwent cytoreductive surgery and chemotherapy with TP at the Department of Gynecologic Oncology in Shandong Cancer Hospital Affiliated to Shandong University. In Table I the baseline patient characteristics are displayed. A total of 14 patients were excluded due to incomplete clinical and pathological information. We divided the patients into a chemotherapy-sensitive group $(n=160)$ and chemotherapy-resistant group $(n=95)$ according to whether or not the tumor relapsed within 6 months after the last chemotherapy. The patients were divided into a low expression group ( $\mathrm{n}=176$, Fig. 5A) and a high expression group $(\mathrm{n}=79$, Fig. 5B) according to the staining of IL-6 protein in interstitial cells. The results revealed that the sensitivity rate of chemotherapy in patients with low expression of IL-6 was $69.3 \%$ and the sensitivity of chemotherapy in patients with high expression of IL-6 was $48.1 \%$. The Chi-square test revealed that there was a significant difference between the two groups $(\mathrm{P}<0.05)$. Univariate and multivariate analyses revealed that age, CA125, interstitial IL-6 expression and cytoreduction satisfaction were closely related to the sensitivity of the TP regimen in ovarian cancer $(\mathrm{P}<0.05$, Table II).

\section{Discussion}

As an important component of solid tumors, CAFs are not a 'spectator', but a positive 'participant' in the whole process of tumorigenesis, development and metastasis, which can affect the response of the tumor to chemotherapy or radiotherapy $(5,18,19)$. Our study focused on the effects of CAF-derived IL-6 on invasion, EMT and paclitaxel resistance of ovarian cancer cells.

It has been confirmed that CAFs secrete high levels of IL-6 (20,21). Osuala et al (22) have confirmed that the paracrine IL-6 signaling between pre-invasive ductal-carcinoma-in-situ (DCIS) cells and stromal CAFs acts as an important factor in the transformation of the progression of DCIS to invasive breast carcinoma (22). Huynh et al have found that CD90 (+) stromal cells may be the main source of IL-6 in T2-T3 CRC tumors, which supports the stemness of tumor cells and inflammatory tumor microenvironment (8). In the present study, we showed that IL-6 was highly expressed in CAFs, and its expression was significantly higher than that in NFs and cancer cells through tissue-specific and cell-specific studies. We, for the first time, found that CAFs were the major source of IL-6 secretion in ovarian cancer.

Previous studies have reported that IL- 6 can promote the proliferation, invasion and stem cell production of cancer cells (23-25). Subramaniam et al have revealed that high levels of IL-6 are secreted from CAFs isolated from human endometrial cancer (EC) tissues, while IL-6 receptors (IL-6R and gp130) are expressed only in EC epithelial cells but not in CAFs, and CAFs can promote EC growth via the activation of IL-6/STAT-3/c-Myc pathway (26). We found that CAF-derived IL-6 may promote the proliferation in OVCAR3 cells. Our results showed that culture supernatant of CAFs significantly upregulated interstitial markers and decreased the expression of epithelium marker in OVCAR3 cells, suggesting that the EMT was significantly enhanced. However, the effect in EMT markers was significantly attenuated by the addition of IL-6 mAb. EMT is often associated with tumor invasion. Our experiment also found that CAF-derived IL- 6 promoted the invasion in ovarian cancer cells. However, NFs' supernatant had no significant effect on the invasion of OVCAR3 cells. Therefore, we, for the first time, found that CAF-derived IL-6 was one of the main factors in the EMT of ovarian cancer.

Xiao et al (27) have found that IL-6 treatment can change the phenotype of human peritoneal mesothelial cells from the typical cobblestone-like to the fibroblast-like appearance in vitro. IL-6 treatment increased the expression of $\alpha$-SMA, but decreased the 
A

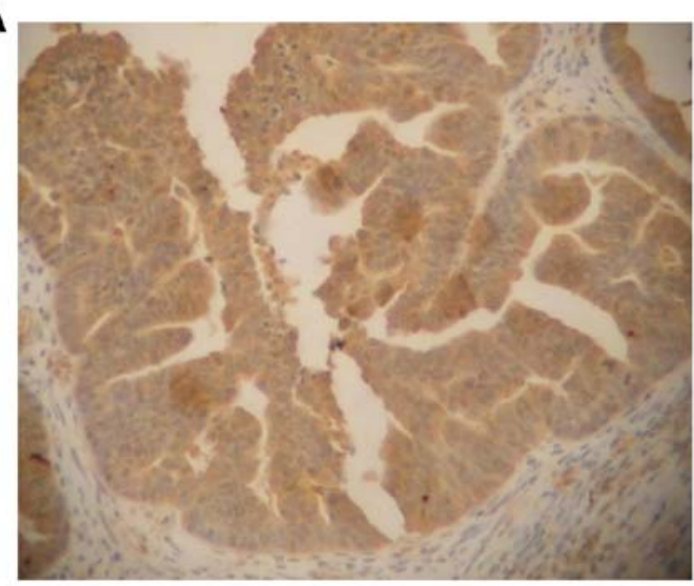

B

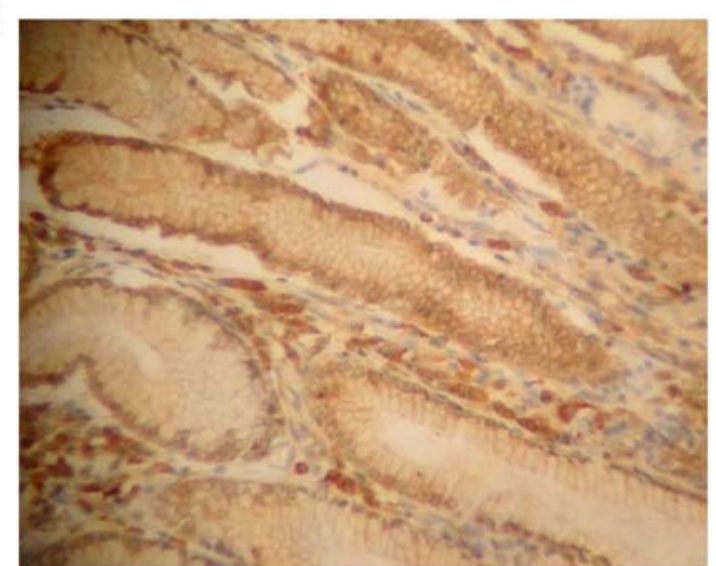

C

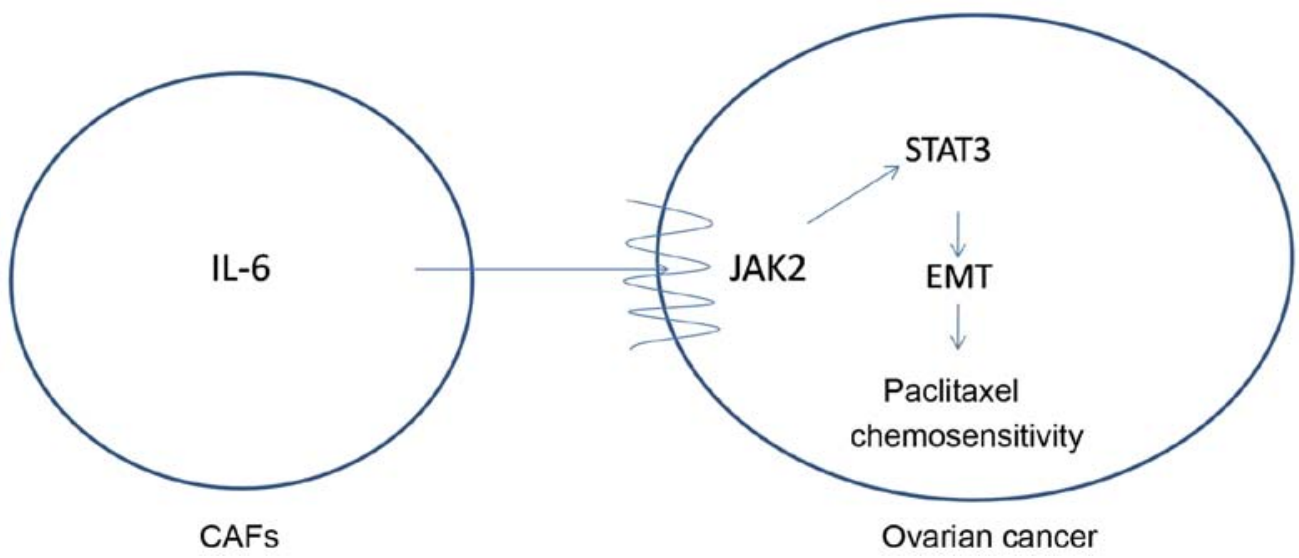

Figure 5. The expression of IL-6 in tumor stroma. (A) Low expression of interstitial IL-6 protein in paraffin-embedded tissues (100-fold). (B) High expression of interstitial IL-6 protein in paraffin-embedded tissues (100-fold). (C) Schematic diagram which demonstrates that CAFs secrete IL-6 and promote $\beta$-TGF-mediated EMT in ovarian cancer cells via the JAK2/STAT3 signaling pathway, leading to the proliferation and invasion of ovarian cancer cells, as well as to apoptosis inhibition and paclitaxel resistance.

Table II. Univariate and binary logistic regression analyses of the association between prognostic factors and chemosensitivity.

\begin{tabular}{lccc}
\hline Factors & $\begin{array}{c}\text { Univariate analysis } \\
\text { HR }(95 \% \mathrm{CI})\end{array}$ & P-value & $\begin{array}{c}\text { Binary logistic regression } \\
\text { analysis HR (95\% CI) }\end{array}$ \\
\hline Age at diagnosis & $10.438(0.229-0.835)$ & 0.012 & $10.368(0.185-0.729)$ \\
Pathological type & $10.962(0.739-1.251)$ & 0.770 & \\
Tumor grade & $10.904(0.676-1.207)$ & 0.493 & \\
Tumor stage & $10.623(0.367-1.056)$ & 0.079 & 0.004 \\
CA125 & $10.464(0.275-0.783)$ & 0.004 & $10.502(0.286-0.882)$ \\
Interstitial IL-6 & $10.410(0.238-0.708)$ & 0.001 & $10.487(0.273-0.868)$ \\
Neoadjuvant chemotherapy & $11.687(0.793-3.592)$ & 0.175 & 0.016 \\
Cytoreduction satisfaction & $10.517(0.300-0.889)$ & 0.017 & 0.015 \\
\hline
\end{tabular}

expression of E-cadherin. IL-6 treatment activates the JAK/STAT signaling pathway, while the JAK2/STAT3 inhibitor prevents IL-6-induced EMT. Wu et al (23) have found that IL-6, secreted by CAFs, promoted EMT and metastasis of gastric cancer via the JAK2/STAT3 signaling pathway. We found that CAF-derived IL-6 activated the JAK2/STAT3 signaling pathway. Following the addition of IL- $6 \mathrm{mAb}$, the phosphorylation levels of JAK2 and STAT3 proteins were decreased. Western blot analysis revealed that the JAK2/STAT3 signaling-pathway-specific inhibitor AG490 prevented IL-6-induced EMT in OVCAR3 cells. This result indicated that CAF-derived IL- 6 mediated the EMT in OVCAR3 cells via the JAK2/STAT3 pathway. IL-6 may alter a series of downstream pathways in ovarian cancer, such as the IL-6/STAT-3/c-Myc pathway in EC, which may also play a role in the EMT of ovarian cancer and will be the aim of our next study. 
EMT has been demonstrated to enhance apoptosis resistance. Shintani et al (28) have demonstrated that CAF-derived IL-6 can cause cisplatin resistance in non-small cell lung cancer (NSCLC) by inducing EMT. The expression of IL-6 in the matrix is an independent prognostic factor for NSCLC (28). Yan et al (29) have found that CAFs can activate the STAT3 signaling, and then decrease cisplatin-induced apoptosis and promote cisplatin resistance in ovarian cancer. There are many ways to acquire tumor chemotherapy resistance, and the present study indicated that EMT was one of the possible pathways for ovarian cancer. The mechanism through which EMT results in chemotherapy resistance in ovarian cancer remains largely unexplored, however recently published studies have indicated the important role of apoptosis resistance $(14,15)$. We investigated the effect of EMT on tumor resistance in the view of apoptosis resistance. The expression of EMT-related proteins in cancer cells were consistent with apoptotic resistance after paclitaxel treatment. By inhibiting EMT with $\beta$-TGF inhibitors, both apoptosis and paclitaxel resistance were decreased. Therefore, we hypothesized that EMT may play an important role in paclitaxel resistance by mediating apoptosis in ovarian cancer.

Paclitaxel combined with carboplatin/cisplatin is the first-line chemotherapy of epithelial ovarian cancer. Tumors, which relapsed within 6 months after the last chemotherapy, could be considered to be relatively resistant to TP regimens, according to platinum-resistance criteria. We divided the patients into a chemotherapy-sensitive group and a chemotherapy-resistant group according to these criteria. IL-6 protein was found to be mainly expressed in the stromal cells and its expression was significantly increased in the drug-resistant ovarian cancer, which was closely related to the sensitivity of TP chemotherapy (Fig. 5A and B). Multivariate analysis revealed that interstitial IL-6 expression was an independent influencing factor of the TP chemotherapy sensitivity (Table II, $\mathrm{P}<0.05)$. Combined with previous experiments, we further confirmed that interstitial IL-6 expression was significantly correlated with paclitaxel resistance. This finding indicated that inhibition of the expression of IL- 6 in the interstitium may be the next step to reverse the targeting of paclitaxel resistance in ovarian cancer.

In conclusion, CAFs could highly secrete IL-6 and promote $\beta$-TGF-mediated EMT in ovarian cancer cells via the JAK2/STAT3 signaling pathway, leading to promoted proliferation and invasion of ovarian cancer cells, as well as inhibited apoptosis and paclitaxel resistance (Fig. 5C; schematic diagram). Therefore, a better understanding of the role of the tumor microenvironment in EMT may facilitate the development of novel therapies for the drug-resistant ovarian cancer.

\section{Acknowledgements}

Not applicable.

\section{Funding}

The present study was supported by the Natural Science Foundation of Shandong Province (grant nos. ZR2017PH029 and ZR2015YL047) and the Projects of Medical and Health Technology Development Program of Shandong Province (grant no. 2015WS0157).

\section{Availability of data and materials}

The datasets used during the current study are available from the corresponding author on reasonable request.

\section{Authors' contributions}

LC designed the research. LW and FZ performed the research and wrote the manuscript. All authors analysed the data and were involved in writing the manuscript. All authors read and approved the manuscript and agree to be accountable for all aspects of the research in ensuring that the accuracy or integrity of any part of the work are appropriately investigated and resolved.

\section{Ethics approval and consent to participate}

Written informed consent was obtained from all of the patients prior to treatment. This study was approved by the ethics committee of Shandong Cancer Hospital Affiliated to Shandong University.

\section{Consent for publication}

Identifying information, including names, initials, date of birth or hospital numbers, images or statements were not included in the manuscript. Written informed consent was obtained from all of the patients prior to treatment.

\section{Competing interests}

The authors declare that they have no competing interests.

\section{References}

1. Siegel RL, Miller KD and Jemal A: Cancer statistics, 2017. CA Cancer J Clin 67: 7-30, 2017.

2. Polyak K and Weinberg RA: Transitions between epithelial and mesenchymal states: Acquisition of malignant and stem cell traits. Nat Rev Cancer 9: 265-273, 2009.

3. Thiery JP, Acloque H, Huang RY and Nieto MA: Epithelial-mesenchymal transitions in development and disease. Cell 139: 871-890, 2009.

4. Erez N, Glanz S, Raz Y, Avivi C and Barshack I: Cancer associated fibroblasts express pro-inflammatory factors in human breast and ovarian tumors. Biochem Biophys Res Commun 437: 397-402. 2013.

5. Del Prete A, Allavena P, Santoro G, Fumarulo R, Corsi MM and Mantovani A: Molecular pathways in cancer-related inflammation. Biochem Med 21: 264-275, 2011.

6. Chen R, Yu Z, Zhang H, Ding J and Chen B: Primary malignant lymphoma of the uterus and broad ligament: A case report and review of literature. Onco Targets Ther 8: 265-268, 2015.

7. Polanska UM and Orimo A: Carcinoma-associated fibroblasts: Non-neoplastic tumour-promoting mesenchymal cells. J Cell Physiol 228: 1651-1657, 2013.

8. Huynh PT, Beswick EJ, Coronado YA, Johnson P, O'Connell MR, Watts T, Singh P, Qiu S, Morris K, Powell DW and Pinchuk IV: CD90 $0^{+}$stromal cells are the major source of IL-6, which supports cancer stem-like cells and inflammation in colorectal cancer. Int J Cancer 138: 1971-1981, 2016.

9. Johansson AC, Ansell A, Jerhammar F, Lindh MB, Grénman R, Munck-Wikland E, Östman A and Roberg K: Cancer-associated fibroblasts induce matrix metalloproteinase-mediated cetuximab resistance in head and neck squamous cell carcinoma cells. Mol Cancer Res 10: 1158-1168, 2012. 
10. Au Yeung CL, Co NN, Tsuruga T, Yeung TL, Kwan SY, Leung CS, Li Y, Lu ES, Kwan K, Wong KK, et al: Exosomal transfer of stroma-derived miR21 confers paclitaxel resistance in ovarian cancer cells through targeting APAF1. Nat Commun 7: 11150, 2016.

11. Abubaker K, Luwor RB, Escalona R, McNally O, Quinn MA, Thompson EW, Findlay JK and Ahmed N: Targeted disruption of the JAK2/STAT3 pathway in combination with systemic administration of paclitaxel inhibits the priming of ovarian cancer stem cells leading to a reduced tumor burden. Front Oncol 4: 75, 2014

12. Colomiere M, Ward AC, Riley C, Trenerry MK, Cameron-Smith D, Findlay J, Ackland L and Ahmed N: Cross talk of signals between EGFR and IL-6R through JAK2/STAT3 mediate epithelial-mesenchymal transition in ovarian carcinomas. Br J Cancer 100: 134-144, 2009.

13. Wu J, Zhang J, Shen B, Yin K, Xu J, Gao W and Zhang L: Long noncoding RNA lncTCF7, induced by IL-6/STAT3 transactivation, promotes hepatocellular carcinoma aggressiveness through epithelial-mesenchymal transition. J Exp Clin Cancer Res 34 $116,2015$.

14. Fischer KR, Durrans A, Lee S, Sheng J, Li F, Wong ST, Choi H, El Rayes T, Ryu S, Troeger J, et al: Epithelial-to-mesenchymal transition is not required for lung metastasis but contributes to chemoresistance. Nature 527: 472-476, 2015.

15. Zheng X, Carstens JL, Kim J, Scheible M, Kaye J, Sugimoto H, Wu CC, LeBleu VS and Kalluri R: Epithelial-to-mesenchymal transition is dispensable for metastasis but induces chemoresistance in pancreatic cancer. Nature 527: 525-530, 2015.

16. Nagasaki T, Hara M, Nakanishi H, Takahashi H, Sato $M$ and Takeyama H: Interleukin-6 released by colon cancer-associated fibroblasts is critical for tumour angiogenesis: Anti-interleukin-6 receptor antibody suppressed angiogenesis and inhibited tumour-stroma interaction. Br J Cancer 110: 469-478, 2014

17. Takai M, Terai Y, Kawaguchi H, Ashihara K, Fujiwara S, Tanaka T, Tsunetoh S, Tanaka Y, Sasaki H, Kanemura M, et al: The EMT (epithelial-mesenchymal-transition)-related protein expression indicates the metastatic status and prognosis in patients with ovarian cancer. J Ovarian Res 7: 76, 2014.

18. Tang X, Hou Y, Yang G, Wang X, Tang S, Du YE, Yang L, Yu T, Zhang H, Zhou M, et al: Stromal miR-200s contribute to breast cancer cell invasion through CAF activation and ECM remodeling. Cell Death Differ 23: 132-145, 2016.

19. Li X, Fan Q, Li J, Song J and Gu Y: MiR-124 down-regulation is critical for cancer associated fibroblasts-enhanced tumor growth of oral carcinoma. Exp Cell Res 351: 100-108, 2017.

20. Dobrzycka B, Mackowiak-Matejczyk B, Terlikowska KM, Kulesza-Bronczyk B, Kinalski M and Terlikowski SJ: Serum levels of IL-6, IL-8 and CRP as prognostic factors in epithelial ovarian cancer. Eur Cytokine Netw 24: 106-113, 2013.
21. Lo CW, Chen MW, Hsiao M, Wang S, Chen CA, Hsiao SM, Chang JS, Lai TC, Rose-John S, Kuo ML and Wei LH: IL-6 trans-signaling in formation and progression of malignant ascites in ovarian cancer. Cancer Res 71: 424-434, 2011.

22. Osuala KO, Sameni M, Shah S, Aggarwal N, Simonait ML, Franco OE, Hong Y, Hayward SW, Behbod F, Mattingly RR and Sloane BF: Il-6 signaling between ductal carcinoma in situ cells and carcinoma-associated fibroblasts mediates tumor cell growth and migration. BMC Cancer 15: 584, 2015.

23. Wu X, Tao P, Zhou Q, Li J, Yu Z, Wang X, Li J, Li C, Yan M, Zhu Z, et al: IL-6 secreted by cancer-associated fibroblasts promotes epithelial-mesenchymal transition and metastasis of gastric cancer via JAK2/STAT3 signaling pathway. Oncotarget 8: 20741-20750, 2017.

24. Wang Y, Li L, Guo X, Jin X, Sun W, Zhang X and Xu RC: Interleukin- 6 signaling regulates anchorage-independent growth, proliferation, adhesion and invasion in human ovarian cancer cells. Cytokine 59: 228-236, 2012.

25. Jin S, Mutvei AP, Chivukula IV, Andersson ER, Ramsköld D, Sandberg R, Lee KL, Kronqvist P, Mamaeva V, Ostling P, et al: Non-canonical Notch signaling activates IL-6/JAK/STAT signaling in breast tumor cells and is controlled by p53 and IKK $\alpha /$ IKK $\beta$. Oncogene 32: 4892-4902, 2013.

26. Subramaniam KS, Omar IS, Kwong SC, Mohamed Z, Woo YL, Mat Adenan NA and Chung I: Cancer-associated fibroblasts promote endometrial cancer growth via activation of interleukin-6/STAT-3/c-Myc pathway. Am J Cancer Res 6: 200-213, 2016

27. Xiao J, Gong Y, Chen Y, Yu D, Wang X, Zhang X, Dou Y, Liu D, Cheng G, Lu S, et al: IL-6 promotes epithelial-to-mesenchymal transition of human peritoneal mesothelial cells possibly through JAK2/STAT3 signaling pathway. Am J Physiol Renal Physiol 313: F310-F318, 2017.

28. Shintani Y, Fujiwara A, Kimura T, Kawamura T, Funaki S, Minami M and Okumura M: IL-6 secreted from cancer-associated fibroblasts mediates chemoresistance in NSCLC by increasing epithelial-mesenchymal transition signaling. J Thorac Oncol 11: 1482-1492, 2016.

29. Yan H, Guo BY and Zhang S: Cancer-associated fibroblasts attenuate Cisplatin-induced apoptosis in ovarian cancer cells by promoting STAT3 signaling. Biochem Biophys Res Commun 470: 947-954, 2016. 